\title{
colaboraciones
}

\section{RESPUESTA DE LA MADERA ANTE EL FUEGO EN LA CONSTRUCCION}

\section{Luis Miguel Elvira Martín}

Dr. Ingeniero de Montes

Jefe del Laboratorio del Fuego

Departamento de Maderas

I.N.I.A.

$660-3$

\section{Sinopsis}

El presente artículo pretende dar una amplia visión del material madera y de los elementos que la utilizan como materia prima (tableros, puertas, etc.), cuando se encuentran sometidos a la acción del fuego.

Se hace un análisis partiendo de la base de realización de ensayos a partir de la Normativa española al respecto, considerando las especies más frecuentemente empleadas en nuestro país, $y$ abarcando los aspectos de duración cortafuegos, estabilidad mecánica sin deformación y estanquidad a las llamas, sin olvidar la emisión de gases inflamables y clasificación frente al fuego.

A través de la experiencia que el Laboratorio del Fuego del INIA posee, y que se exponen en esta colaboración, se pueden extraer consecuencias que permitan la utilización de la madera en construcción de una forma más lógica, eliminando algunos prejuicios que existen sobre su naturaleza y comportamiento frente al fuego.

\section{CONCEPTOS BASICOS, INCENDIO, REACCION Y RESISTENCIA AL FUEGO}

Un incendio es un fuego incontrolado cuya magnitud es consecuencia de los materiales que se encuentran en el edificio y cuya duración es función de los elementos estructurales con que dicho edificio esté construido. De ello se deduce que está condicionado a la calidad frente al fuego de todos los productos y estructuras que lo forman, considerados en conjunto.

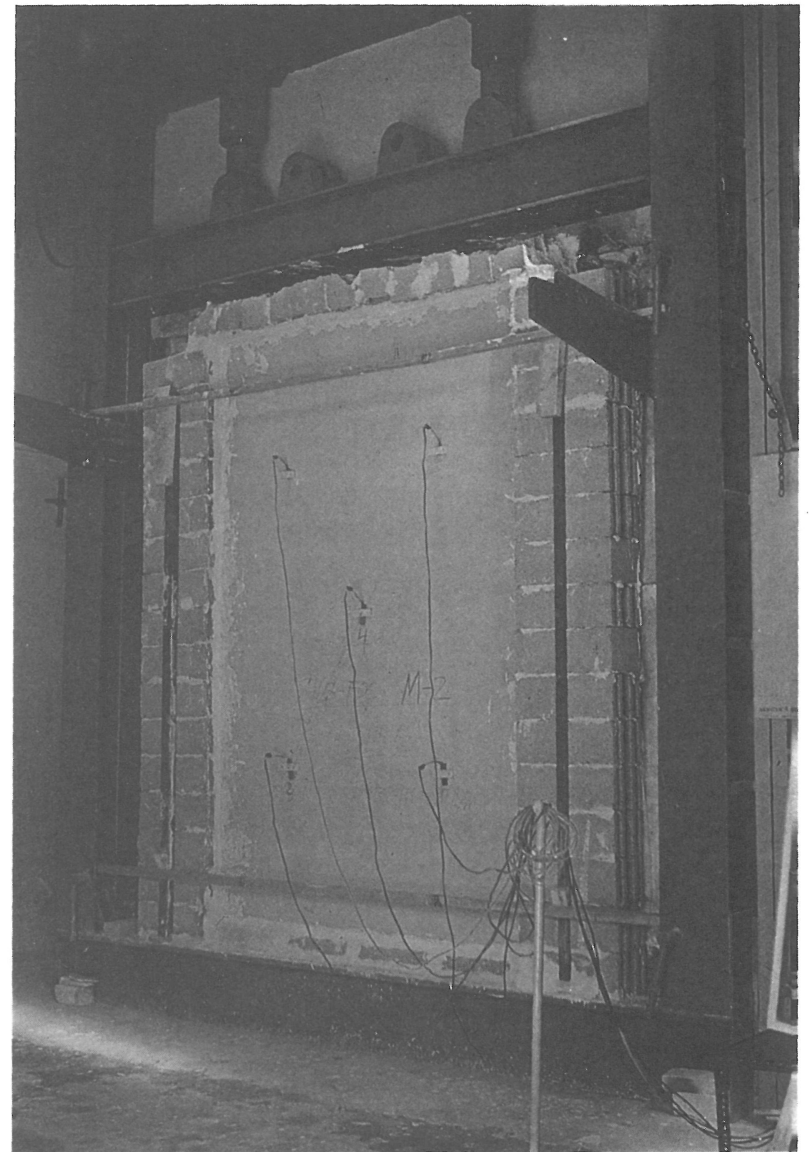

Tablero de particulas de madera antes del ensayo de resistencia al fuego.

La reacción al fuego de cada material es el alimento que puede aportar al fuego y a su desarroIlo. De ella dependen directamente la virulencia del incendio, su evolución, el pánico en los habitantes, etc.

La resistencia al fuego de una estructura es el tiempo que ésta se mantiene cumpliendo su función al ser atacada por el fuego. Representa el tiempo de que se dispone para huida y salvamento de bienes en razón a esa estructura.

El grado de reacción y de resistencia al fuego de un material o estructura miden su comportamiento al fuego, siendo el incendio el conjunto de los de cuantos materiales y estructuras existan afectadas por aquél.

La reacción al fuego es importante al principio del incendio. Si es alta éste se iniciará fácilmente y se desarrollará de forma rápida. Habrá que redu- 


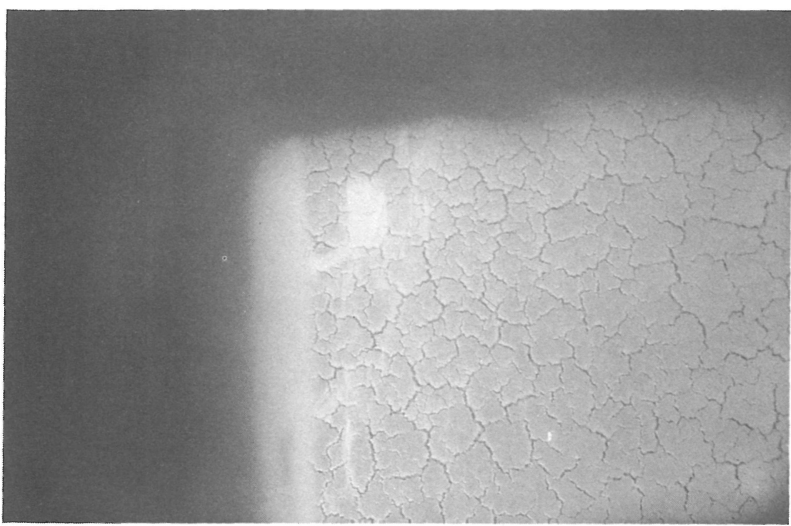

Tablero de particulas durante el ensayo. Cese de la inflamación y formación de capa prctectora de carbón.

cirla en este caso mediante ignifugantes o evitando la colocación de materiales tan reactivos.

En la fase de desarrollo del incendio influyen tanto la reacción como la resistencia, pues aquella violencia puede ser frenada por la existencia de comportamientos estancos al fuego construidos con estructuras de alta resistencia al mismo, puertas y tabiques cortafuegos, etc.

En la fase final las estructuras propias del edificio evitarán su derrumbamiento si son muy resistentes al fuego.

\section{COMPORTAMIENTO AL FUEGO DE LA MADERA, SUS DERIVADOS Y SUS ESTRUCTURAS}

La reacción al fuego de un material y también por tanto la de la madera se mide mediante la norma UNE-23-103-73 y que determina si el material es combustible o no, dándole tal clasificación; es decir, si es capaz de mantener fácilmente reacción química con el oxígeno.

Si efectivamente es combustible ( $y$ en este caso se encuentra la madera), puede ser más o menos inflamable, por tardar mucho tiempo o poco en iniciarse la llama, desprendiendo calor abundante, llama larga o corta, etc. En este sentido se clasificará en 5 clases, desde $M-1$ a $M-5$ en inflamabilidad creciente y de acuerdo con la Norma UNE 23-721.

En cuanto a la resistencia al fuego se utilizan las normas UNE-23.093 para estructuras en general y las UNE-23-802 para aquellos que cierren huecos (puertas, ventanas, tabiques, etc.) o la UNE 23.803 para estructuras vidriadas.

El comportamiento al fuego de la madera se recoge, comparado con el de otras estructuras, en nuestra obra "Comportamiento al fuego de mate- riales y estructuras" y su utilización específica en "Criterios de utilización de la madera frente al fuego" de próxima publicación, o bien en la norma DIN-4102 Parte 4, pero tal comportamiento puede resumirse como sigue.

\subsection{LA MADERA MACIZA}

\subsubsection{Reacción al fuego}

Está fundamentalmente relacionada con su constitución en forma de tubos (vasos o fibras) y con el contenido de humedad.

\subsubsection{Combustión}

Cuando se efectúa en exceso de aire la temperatura de la llama es función de la humedad. Así por ejemplo, en una madera al $15 \%$ de humedad, se tiene una temperatura en llama de $1.150^{\circ} \mathrm{C}$. Si la madera es anhidra, se alcanzan hasta $1.800{ }^{\circ} \mathrm{C}$ en la llama. En cualquier caso, la temperatura de Ilama supera los $1.000{ }^{\circ} \mathrm{C}$. Ello da a la llama un poder de propagación proporcional a su temperatura.

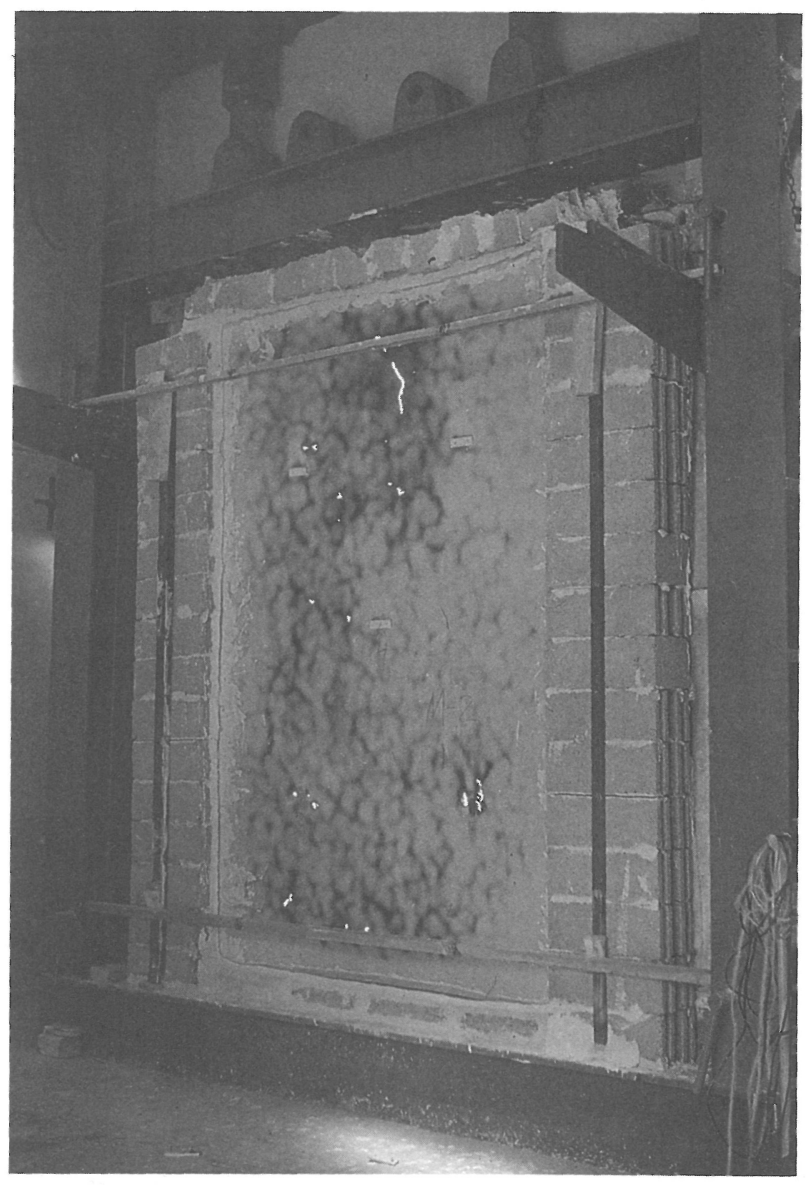

Tablero de particulas. Fin del ensayo con la misma duración CORTAFUEGOS, PARALLAMAS y ESTABLE al fuego. Ausencia de deformaciones, estabilidad de la estructura. Aislamiento térmico hasta el final. 
Mientras tanto, la madera se encuentra a una temperatura de 400 a $600{ }^{\circ} \mathrm{C}$ por debajo de los cuales no se mantiene ya la combustión viva.

Esto puede ocurrir con exceso o también con defecto de aire. Tal es el caso de una viga gruesa: el carbón superficial hace la combustión interna con defecto de aire, el calor se disipa por convección, la madera en el interior de la viga está a menos de $275^{\circ} \mathrm{C}$ y se apaga.

Si la combustión tiene lugar con defecto de aire se desprenden gases en diferentes proporciones $\mathrm{CO}_{2}, \mathrm{CO}, \mathrm{H}_{2}$ e hidrocarburos.

En una primera fase endotérmica, hasta los $150{ }^{\circ} \mathrm{C}$, la madera absorbe calor que sólo emplea en evaporar el agua y secarse.

De 150 a $280{ }^{\circ} \mathrm{C}$ continúa absorbiendo calor y desprende gases alcohólicos y ácidos formados por un $30 \%$ de CO (combustible) y un $70 \%$ de $\mathrm{CO}_{2}$ (incombustible). La madera presenta, en esta fase, un color marrón.

De 280 a $380{ }^{\circ} \mathrm{C}$ la combustión se hace exotérmica, la madera desprende calor y gases abundan-

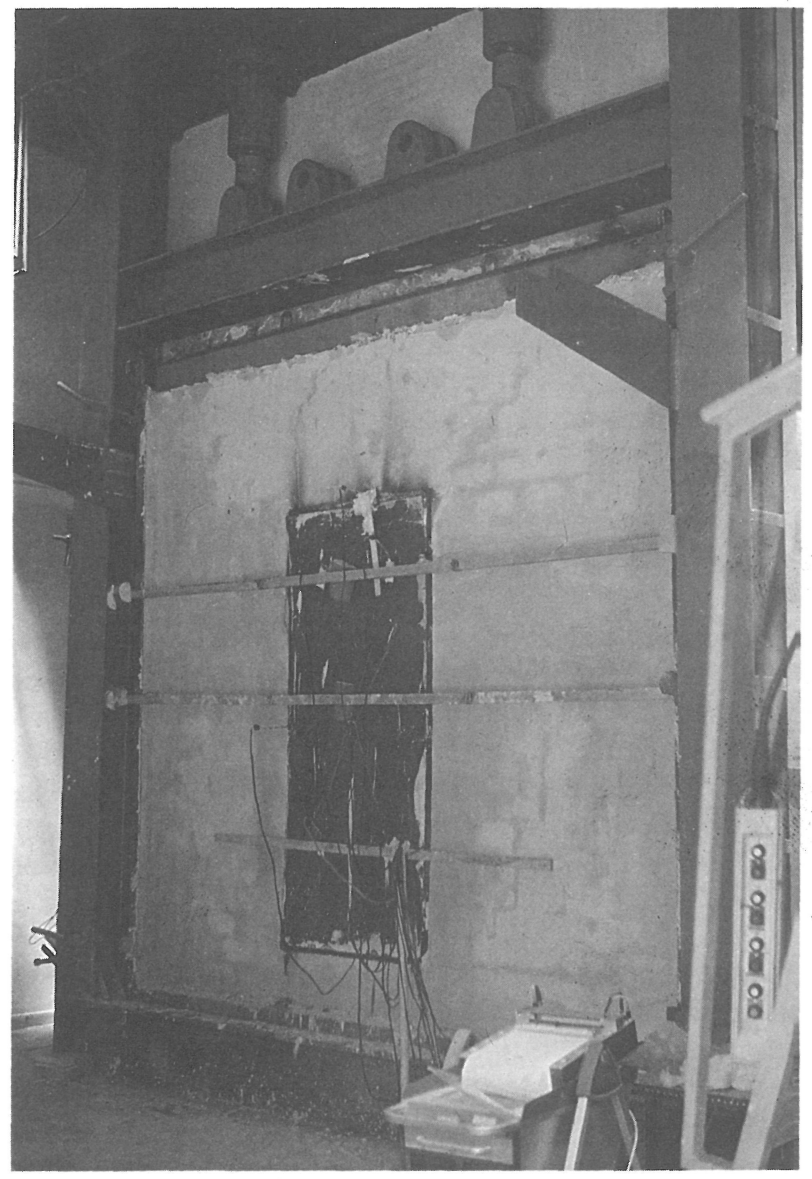

Resistencia al fuego de una puerta metálica. Rápidas deformaciones. Pérdida de estanqueidad. Calentamiento en la cara no expuesta.

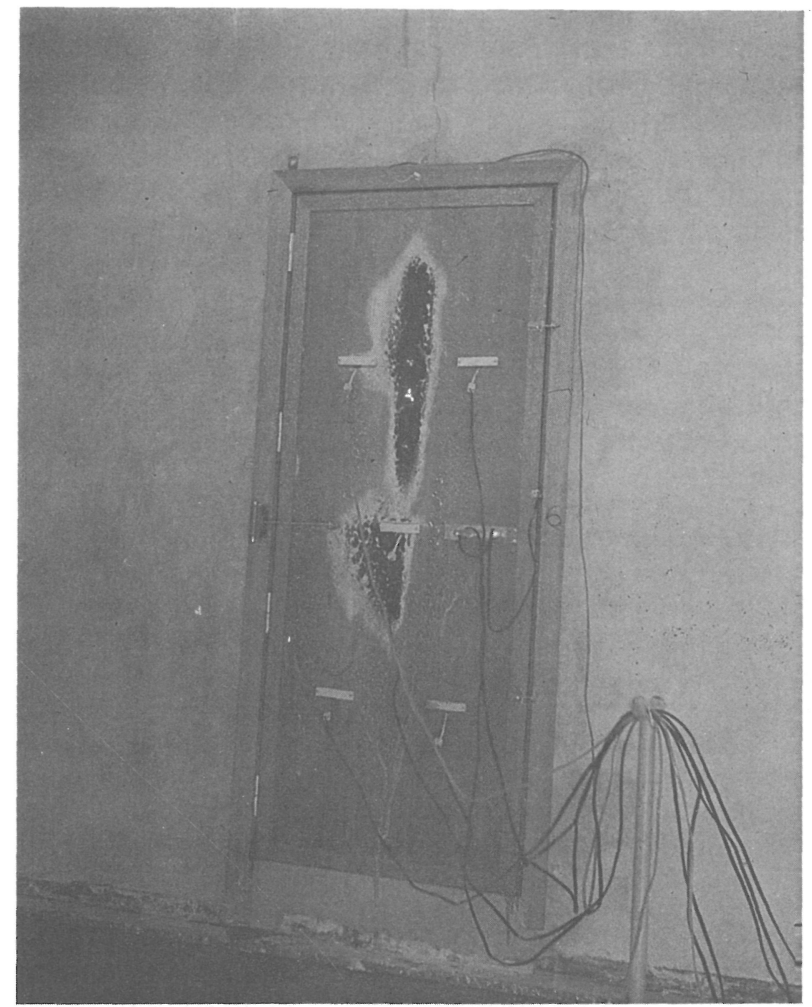

Resistencia al fuego de una puerta de madera. Estanqueidad hasta el final del ensayo. Estabilidad total sin deformaciones. Aislamiento térmico hasta el final del ensayo.

tes, apareciendo los hidrocarburos y disminuyendo el $\mathrm{CO}_{2}$. Presenta un color negro achocolatado.

Hacia los $350^{\circ} \mathrm{C}$ todos los gases que se desprenden son combustibles, aunque escasos y abundan los hidrocarburos.

Por encima de los $450{ }^{\circ} \mathrm{C}$ están presentes el hidrógeno y los hidrocarburos y un residuo de carbón inflamable él mismo.

\subsubsection{Poder calorífico}

El poder calorífico de las fibras de la madera es prácticamente constante, pero las materias contenidas en aquéllas, resinas, taninos y materias extractivas, aumentan o disminuyen dicho poder calorífico. Así, por ejemplo, la resina tiene un poder calorífico de 9.000 a $10.000 \mathrm{cal} / \mathrm{gr}$. (37,7 MJ/kp a $41,9 \mathrm{MJ} / \mathrm{kp}$ ) y la lignina $6.000 \mathrm{cal} / \mathrm{gr}$.

Según la proporción en que intervienen en la madera, así varía el poder calorífico de ésta, tomando valores comprendidos entre los límites de 2.800 y $5.000 \mathrm{cal} / \mathrm{gr}$. y admitiéndose como valor medio $16,76 \mathrm{MJ} / \mathrm{kp}(4.000 \mathrm{Kcal} / \mathrm{kg})$.

Otro factor que modifica el poder calorífico es la humedad contenida en la madera, pues como sabemos está en equilibrio con las condiciones ambientales. Este factor, además influirá en todo el 
66

Informes de la Construcción. Vol. $35, n,{ }^{\circ} 358$, marzo, 1984

proceso de reacción y resistencla al ruego $y$, por lo tanto, es preciso citar la humedad a que se encuentra. Asi, $1 \mathrm{~kg}$. de madera al $12 \%$ de hum medad tiene un poder calorifico superior, igual al de 900 gr. de madera seca. Por todo ello, se com prende que el poder calorifico de una misma especie es variable incluso si la madera es anhidra.

Las maderas densas tienen más p.c.\$. que las Iigeras, pero sl éstas tienen, como es frecuente, sustancias extractivas, ellas elevan a aquél y en definitiva pueden tener p.c. parecido; sin embargo, la madera densa arderá más despacio.

\subsubsection{Inflamabilliad}

La inflamabllidad es la facllidad que tiene un combustible para poder emitir gases que se inflamen. Es evidentemente función de:

1. De la posibilidad de que estos gases puedan salir al exterior al aportar calor.

2. Del flujo del calor que es necesario aportar al material para que esto ocurra.

3. Del punto de inflamación del material, enten diendose por tal la temperatura a la cual el combustible emite gases capaces de inilamarse con una chispa o llama.

En el caso de la madera, estos gases son hidrom carburos en su mayoría.

Se admite como punto de inflamación los $275^{\circ} \mathrm{C}$, sin embargo, no es fijo, pesando fuertemente la especie.

Al principlo de la combustión el calor aportado a la madera se emplea en evaporar ol agua de la misma, produciéndose un efecto de secado, no teniendo lugar la combustión hasta que aquella esté seca. Con ello se reduce el loco calorifico en la primera fase. El efecto es el mismo que sl se arrojase agua al fuego. De aqui se deduce que raras veces es la madera la causa de la iniciación del fuego, salvo en almacenes de serrin, vigas muy secas o en alguna otra ocasión excepcional.

De la misma forma actúa la humedad ambiente, pues ya sabemos que cada especie tiene un contenido de humedad, en equilibrio con el de la atmosfera y aquélla hace incombustible a la made. ra, empleando el calor recibido en evaporar el contenido de agua en vez de producir calor.

La especle de madera influye fuertemente en la inflamabilidad. Las especies más ligeras al contener un volumen grande de poros, ofrecen gran II bertad a los gases para su desprendimiento. Dl. chos gases, en su mayoria inflamables, provocan rápldamente las llamas. Por ello, ol roble se infla. ma menos que el abeto o el chopo.

La forma de emplear la madera, ol estado del mam terial y la humedad ambiente son también factores decisivos para la inflamación. Asi, por ejemplo, un parquet tendrá distinta inflamación según el tipo de solera sobre el que esté colocado, el tipo de cola, etc. La superficle rugosa y con algunos ángulos agudos, la favorecen; un caso extremo es el del serrin (superl/Vol grande) o polvo de madera, en el cual la inflamación se efectúa en cadena y da lugar a una explosión. El caso opuesto es el de las vigas en que por ser elementos gruesos la inflamación es supericial.

Los taninos y resinas además de aumentar el poder calorifico, aumentan la inflamabilidad de la madera y en conjunto todas estas variables citam das hacen dificll de predecir y comparar la Inflam mabilidad de las distintas especies, hablando de forma general.

La madera es, por tanto, combustible e infla. mable en estado natural, pero puede eliminarse esta inflamación totalmente, mediante tratamien los ignifugos, teniéndose en definitiva un material de construcción seguro ante el fuego.

\subsubsection{Humos y gases noclvos}

La velocidad de combustión y, por tanto, de desprendimiento de los gases tóxicos es el factor que hace éstos extremadamente peligrosos y caum santes do victimas. En la madera, la velocidad do combustión es lenta y el desprendimiento de $\mathrm{CO}_{2}$ y CO es proporcional a la temperatura, admitiendose que los gases, aunque intervenga el CO y $\mathrm{CO}_{2}$, son poco pellgrosos.

Otros materlales, como los plásticos halogenados, poseen productos de pirólisis o de combustion, análogos a los de ofros materiales tradicionales como la madera, pero la velocidad de combustión es rapidisima, por 10 que con estos materiales on poco tiempo hay un gran desprendimiento de ca. lor, un empobrecimiento de oxigeno y se despren. den los gases tóxicos antes que en los incendios tradicionales o en los que existe madera. El ambiente letal, la perdida de conocimiento y el enve. nenamiento sobrevienen mucho antes.

Si, además, como on los plásticos clorados (PVC), se desprende en la combustión el ácido clorhidrico con un doble efecto, acción corrosiva y excilante pulmonar provocador de hemorragias $y$ en otros ol ácido cianhidrico (CNH), también corrosivo, hace, con razón, a estos productos plásti. cos unos de los más discutidos en su empleo ante el fuego.

Se comprende fácllmente que una noma de toxi. cidad que sólo valore ol porcentaje o composición química de los humos, no es valida en absoluto: debe considerar también la velocidad de despren. dimiento de los mismos, de lo contrarlo, llevaria a ensayos con resultados aproximados en los incon. dios con materiales clasicos y totamente falsos en aquéllos donde al combustible sea materia plástica, que modemamente son lan irecuentes. 
CUADRO 1

Poder calorifico inferior do la madora anhidra do algunas especies

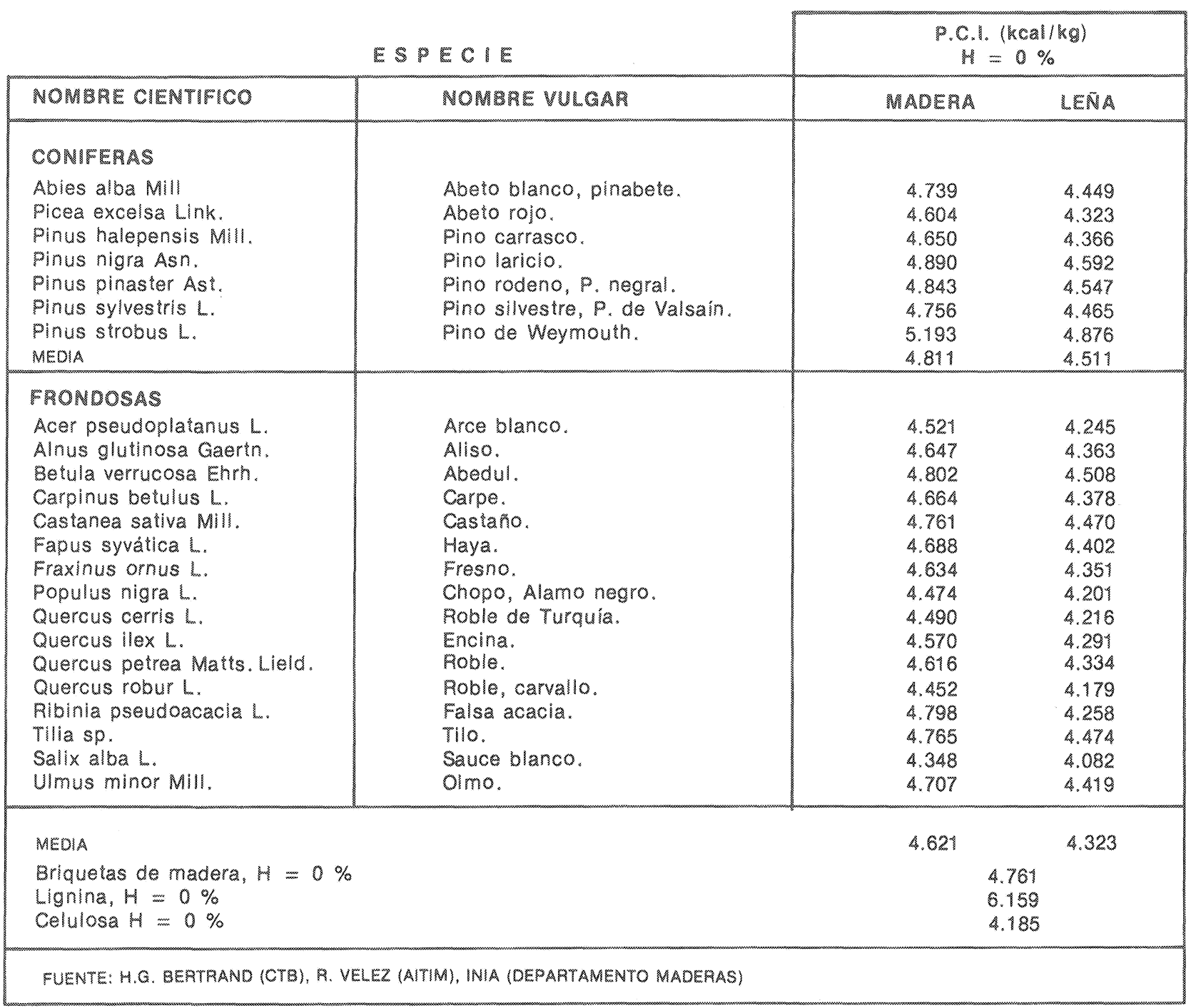

CUADRO 2

Reacción al luego de maderas comerciales nacionales sin ignilugar

\begin{tabular}{|c|c|c|c|c|c|c|c|c|c|c|c|}
\hline \multirow{2}{*}{$N^{0}$} & \multirow{2}{*}{ Especie } & \multicolumn{4}{|c|}{ Indíces } & \multirow{2}{*}{$\begin{array}{c}\text { Clasim } \\
\text { hica- } \\
\text { ción }\end{array}$} & \multirow{2}{*}{$\underset{\%}{\text { Humed. }}$} & \multirow{2}{*}{$\mid \begin{array}{c}\text { Frondosa } \\
y \\
\text { Conitera }\end{array}$} & \multirow{2}{*}{$\begin{array}{c}\text { Espesor } \\
\text { mm }\end{array}$} & \multirow{2}{*}{$\begin{array}{l}\text { Tabla } \\
0 \\
\text { Tabión }\end{array}$} & \multirow{2}{*}{ Observaciones } \\
\hline & & & 1 & s h & $c$ & & & & & & \\
\hline 1 & $\begin{array}{l}\text { Pinus Pinaster Art. } \\
\text { (Pino rodeno. P. negral) }\end{array}$ & 2,3 & 4,25 & 1,5 & 4,77 & $M-4$ & 11,5 & Conitera & 27 & Tabla & No se extingue \\
\hline 2 & $\begin{array}{l}\text { Pino sylvestre } L \text {. } \\
\text { (Pino silvestre) }\end{array}$ & 0,92 & 324,46 & 61,8 & 14,8 & $M-4$ & 14,4 & Conifera & 67 & Tablon & No se extingue \\
\hline 3 & $\begin{array}{l}\text { Fagus slavática L } \\
\text { (Haya) }\end{array}$ & 0,96 & 65,06 & 1,5 & 5,58 & $M-4$ & 11,0 & Frondosa & 40 & Tablon & No se extingue \\
\hline 4 & $\begin{array}{l}\text { Fagus sylvática L. } \\
\text { (Haya) }\end{array}$ & 1,1 & 5,55 & 1,8 & 7,53 & $M-4$ & 11,2 & Frondosa & 27 & Tabla & No se extingue \\
\hline 5 & $\begin{array}{l}\text { Quercus Robur L. } \\
\text { (Roble, carvallo) }\end{array}$ & 1,1 & 2,55 & 1,3 & 2,16 & $\mathrm{M}-3$ & 17,8 & Frondosa & 70 & Tablon & \\
\hline 6 & $\begin{array}{l}\text { Populus sp. } \\
\text { (chopo) }\end{array}$ & 2,8 & 4,15 & 61,8 & 7,77 & $M-4$ & 12,8 & Frondosa & 70 & Tablon & No se extingue \\
\hline
\end{tabular}

FUENTE: INIA (DEPARTAMENTO MADERAS) 


\subsubsection{Resistencia al luego}

\subsubsection{Estabilidad mecánica}

La madera sin ignifugar hemos visto que tiene mala reacción al fuego, aunque los ignifugantes la reducen. Sin embargo, tiene una alta resistencia al fuego, pudiendo contener el incendio y mante. ner en ple las partes del edificio mientras se desaloja. Con razón, si bien es el combustible más antiguo, también lo es como material de construcción. Este efecto lo conocen perfectamente los bomberos y prefieren actuar en incendios de edificios con estructura de madera a los que la tienen de hierro u hormigón. La razón de ello estriba, principalmente, en las sigulentes propiedades de la madera:

1. El coeficiente de conductibilidad calorifica de las fibras es muy pequeño y la transmisión de calor al interior de la madera es cada vez más lento y dificil en profundidad.

2. La madera es un material higroscópico. El agua contenida en la misma absorbe calor de evaporación y ésta no entra en combustión hasta que está seca. El tiempo que se emplea en ello es tiempo que se gana en resistencia al fuego.

3. El espesor de las plezas o elementos es otro factor favorable. En las vigas o elementos gruesos la madera comienza ardiendo superficialmente después de haber ganado tiempo hasta su secado. El carbón formado en la su. perficie sirve de protección a la parte interna que dada la baja conductibilidad térmica permanece intacta.

En estas condiciones se produce una disminución de la resistencia mecánica de la pieza por haberse reducido la sección útll, pero como por otra parte la madera seca gana en resistencia mecánica, este último aumento es superior ligeramente a la perdi. da por sección, generalmente.

\subsubsection{Estanqueidad las llamas}

La estanqueidad a las llamas presenta una gran variabilidad, según los materiales de que se trate, incluso en la madera, ofreciendo valores muy al tos en algunas maderas sin ignifugar.

El fallo de la resistencia al fuego por estanqueidad de los elementos de madera, se pone de manitiesto en aquellos elementos de cierre de huecos (puertas, ventanas, etc.).

Se admite que la velocidad de penetración del fue. go en la madera es de $0,7 \mathrm{~mm} / \mathrm{min}$., desoreciando los 3 primeros mm de formación de carbón.

\subsubsection{Emisión de gases inflamables}

Aunque la madera hemos visto que emite gases inflamables (hidrocarburos), lo hace por la cara expuesta, ya que la transmisión de calor a la cara externa es muy débil. No suele, por tanto, observarse este electo.

\subsubsection{Aislamiento térmico}

En el caso que nos ocupa de la madera, al ser baja la conductibilldad térmica, el fuego en la cara expuesta no suele ser capaz de calentar la otra cara. Se tiene, pues, un excelente aislante que facilita la lucha contra el incendio.

\section{CUADRO 3}

Comparación de la resistencia al fuego de pllares de roble y de acero (Ensayos del Centre Technique du Bois)

\begin{tabular}{|c|c|c|}
\hline \multirow{2}{*}{ MATERIAL } & \multicolumn{2}{|c|}{$\begin{array}{c}\text { Tiempo de resistencia al iuego } \\
\text { Carga } 10\end{array}$} \\
\hline & $\begin{array}{c}\text { Plares roble } \\
\text { de } \\
15 \times 15 \times 230 \mathrm{~cm}\end{array}$ & $\begin{array}{c}\text { Pllares acero } \\
\text { de } \\
15 \times 15 \times 230 \mathrm{~cm}\end{array}$ \\
\hline 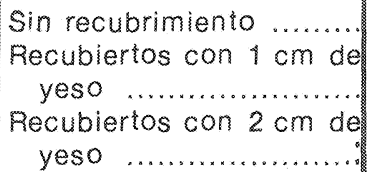 & $\begin{array}{l}52 \text { minutos } \\
81 \text { minutos } \\
118 \text { minutos }\end{array}$ & $\begin{array}{l}8-10 \text { minutos } \\
60-69 \text { minutos } \\
84-95 \text { minutos }\end{array}$ \\
\hline
\end{tabular}

\section{CUADRO}

Rosistonch al luego do dirrontos aspocies do maders (no ignilugadas)

\begin{tabular}{|c|c|}
\hline (Nombre clentinge) & (Nombre vulgar) \\
\hline $\begin{array}{l}\text { Resistencla a suego muy } \\
\text { elevada: }\end{array}$ & \\
\hline $\begin{array}{l}\text { Eucalyptus diversicolor F.V.M. } \\
\text { Eucalyptus marginata Sm. } \\
\text { Mora excelsa Benth. } \\
\text { Ocotea rodiacl Mez. } \\
\text { Tectona grandis L.f. } \\
\text { Terminalia obovata Standl. }\end{array}$ & $\begin{array}{l}\text { Kerri. } \\
\text { Jarrah. } \\
\text { Mora. } \\
\text { Greenhor. } \\
\text { Teca. } \\
\text { Palo amarillo. }\end{array}$ \\
\hline $\begin{array}{l}\text { Resistercia al tueqo } \\
\text { revada: }\end{array}$ & \\
\hline $\begin{array}{l}\text { Acer psudoplatanus } L \text {. } \\
\text { Carpinus betulus } L \text {. } \\
\text { Castanea sativa Mill. } \\
\text { Fagus sylvática } L . \\
\text { Fraxinus excelsior } L . \\
\text { Pinus pinaster Ait. } \\
\text { Quercus cerris L. } \\
\text { Quercus robur L. } \\
\text { Robinia pseudoacacia L. } \\
\text { Taxus baccata L. }\end{array}$ & $\begin{array}{l}\text { Arce blanco. } \\
\text { Carpe. } \\
\text { Castaño. } \\
\text { Haya. } \\
\text { Fresno. } \\
\text { Pino rodeno, P. negra } \\
\text { Roble de Turquia. } \\
\text { Roble, Carvallo. } \\
\text { Falsa acacla. } \\
\text { Telo. }\end{array}$ \\
\hline
\end{tabular}




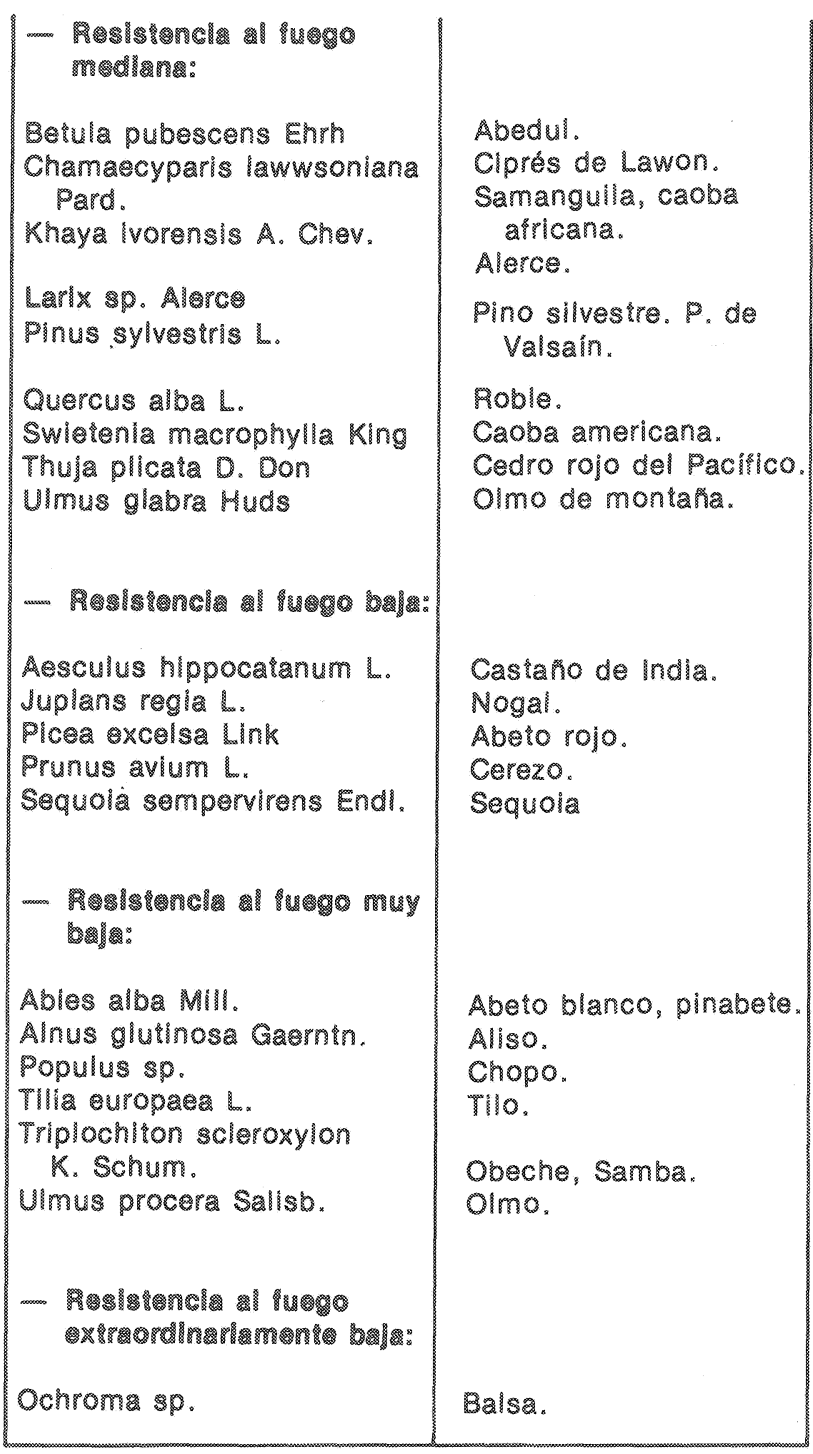

\subsection{EL TABLERO CONTRACHAPADO Y MADERA LAMINADA}

Los tableros contrachapados ofrecen dificultad al paso del calor por la diferente constitución de las capas de madera y proporcionan una resistencia al fuego buena, incluso a veces mayor que la de la madera maciza, siendo función del grosor y del tipo de cola.

Al ser atacada la madera encolada por el fuego, puede producirse un desencolado que divida el material y facilite la propagación y penetración de las llamas, si la cola no es termoestable. Por el contrario, la colla de resorcina formol proporciona una alta resistencia al fuego por mantener unidas las partes quemadas.

Los tableros que emplean colas de contacto se separan en sus elementos integrantes a bajas temperaturas, facilitando a la vez la propagación de las llamas.
Sin embargo, clertas colas de contacto fabricadas a base de caucho sintético o natural, son capaces de recibir endurecedores que aumentan la temperatura de desencolado y con ello la resistencia al fuego de elementos que las emplean.

Con mucho aventajan a éstas las citadas colas de resorcina, que mantienen unido el carbón a la capa de madera no quemada. Este actúa de prom tector como en la madera maciza.

Además del tipo de cola, las técnicas de secado y encolado de la madera, las cargas utilizadas y la dosificación de cola influyen en la resistencia al fuego de este tipo de tablero.

A continuación exponemos los Cuadros 5 y 6 , que corresponden a experiencias efectuadas por el CTB de Paris con colas que, junto con las citadas anteriormente, también pueden emplearse con éxito ante el ruego.

\section{CUADRO 5}

Comparación de los alecios del luego, después de 35 min. en madera laminada y madera maciza (Centre Techniquo du Bois)

\begin{tabular}{|c|c|c|c|}
\hline Materialos & $\begin{array}{l}\text { Grosór } \\
\text { de las } \\
\text { capas }\end{array}$ & Cola & $\begin{array}{l}\text { \% de la } \\
\text { sección } \\
\text { destruldo } \\
\text { por ol } \\
\text { Tuego }\end{array}$ \\
\hline $\begin{array}{c}\text { Madera maciza } \\
\text { (Testigo) }\end{array}$ & & & $52 \%$ \\
\hline Madera laminada & $10 \mathrm{~mm}$ & Resorcina & $49 \%$ \\
\hline Madera laminada & $10 \mathrm{~mm}$ & Caseina & $63 \%$ \\
\hline Madera laminada & $10 \mathrm{~mm}$ & Urea formol & $63 \%$ \\
\hline Madera laminada & $17 \mathrm{~mm}$ & Urea formol & $54 \%$ \\
\hline
\end{tabular}

\section{CUADRO 6}

Resistencia al Puego de los tableros contrachapados según su grosor y según ll tipo de cola

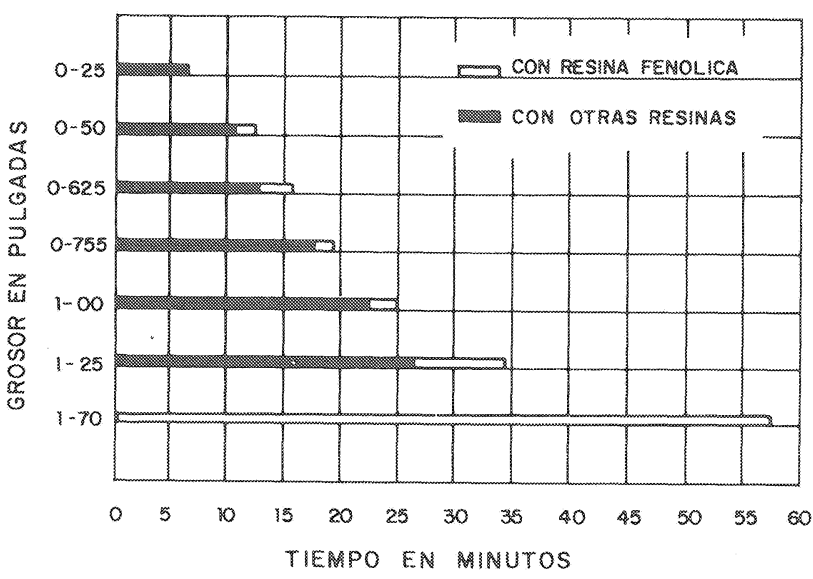


Estos tableros, asi como los de particulas, son ideales para la construcción de tabiques y muros, por la ausencia y reducción de juntas y por su regularidad de carbonización, pudiéndose combinar con cemento, amianto y materiales diversos.

El tablero contrachapado se utiliza también combinado con diversos tipos de resinas para su uso en pavimentos, presentando una gran dureza y resis. tencia a la fricción.

La velocidad de perforación es, trecuentemente, de $0.55 \mathrm{~mm} / \mathrm{min}$. inferior a la de la madera $(0,7 \mathrm{~mm} / \mathrm{min}$. $)$.

\subsection{EL TABLERO AGLOMERADO DE PARTICULAS}

\subsubsection{Reacción al uego}

Los tableros de particulas están constituldos por trozos de madera de muy poco espesor, procedentes de la rragmentación de la misma, secados hasta una cierta humedad, generalmente bala y encolados por pulverización de cola de resina sintética. La mezcla obtenida se prensa a alta temperatura, que asegura la polimerización de las resinas termoendurecibles. En el proceso de fabrica ción pueden introducirse distintos adilivos y pro ductos ignifugantes, consiguiéndose un tratamiento profundo y duradero. Pueden ignifugarse tam. bién por aplicaciones en superificie, pero en este caso debe renovarse el tratamiento periódica. mente.

Se trata, pues, de un material muy elaborado y homogéneo, muy adecuado a todos los usos de la construcción, con caracteristicas frente al fuego muy similares a las de la madera maciza y a veces superiores, pues presentan la ventaja sobre ésta de formar plezas de gran superficie, con ausencia de juntas, circunstancia ésta, que es importante para asegurar una resistencla al luego alta.

Su poder calorifico es ligeramente inferior al de la madera y menor aún en los tableros ignifugados. Hemos obtenido $4.101 \mathrm{kcal} / \mathrm{kg}$ para tablero comercial normal de clase $\mathrm{M}-4$ y para el tablero ignifigudo de clase $\mathrm{Mm}, 3.409 \mathrm{kcal} / \mathrm{kg}$, llegándose a $2.924 \mathrm{kcal} / \mathrm{kg}$ para el tablero $\mathrm{M}-1$ de resistencia mecánica normal.

La clasificación según Norma UNE-23.103 le situa como COMBUSTIBLE incluso si está Ignifugado, pudiendo, en este caso, ser M-1 NO INFLA MABLE.

El espesor del tablero intuye no sólo en su resis. tencia al fuego como en otros materiales, sino en su reacción por ser un derivado de la madera y por regla general se considera que espesores infe- riores a $14 \mathrm{~mm}$ se obtiene clasificación $\mathrm{M}-4$ en tableros sin ignifugar, siendo generalmente de clase $\mathrm{M}-3$ los de espesores superiores a $14 \mathrm{~mm}$.

Los tableros ignifugados en su masa obtienen cla sificaciones $M-1$ y $M-2$, según la intensidad y tipo de tratamiento.

La Ignifugación en masa puede no alterar las propiedades del tablero. Tampoco deben alterarse su humedad, ni la presentación de la superficie, dándole una duración de 10 anos al poder ignifugante.

La ignifugación artificial, con pinturas y barnices ignífugos, permiten seguir manteniendo la clasill. cación inicial del tablero o incluso mejorarla dándole generalmente una validez de ignifugación de 3 anos.

Los tableros de más de $14 \mathrm{~mm}$ con distintos acabados plásticos ofrecen reacciones de $\mathrm{M}-4$, dando también Mu4 sin el recubrimiento. Lo que si he. mos encontrado es que los recubrimientos aumentan ligeramente la resistencia al fuego del tablero base.

Los gases y humos desprendidos son muy volátiles, poco tóxicos y presentan la ventaja, por ello, de servir de detectores del incendio, de tal forma que el tablero de particulas, sl se respetan las normas adecuadás de puesta en obra e ignifuga. ción, no es causante de origen $n$ i extensión del incendio. Muchas veces, las victimas en los incendios no deben atribuirse a los materiales, sino al incorrecto comportamiento de las personas frente al fuego.

Debido a los espesores relativamente gruesos, estos tablero de particulas suelen emplearse in $\mathrm{ig}$ nffugar y solamente en casos especiales, cuando se exigen prescripciones severas, se usan de cla. ses $\mathrm{M}-1$ y $\mathrm{M}-2$.

\subsubsection{Resistencia al fuego}

La estabilidad mecánica es excelente, el tablero se alabea poco y va perdiendo sus particulas a medida que es atacado por el fuego, pero sus va. riaciones dimensionales son muy pequeñas.

El paso de la llama es regular, con un avance que se calcula en $0,7 \mathrm{~mm} / \mathrm{min}$. como en la madera.

Respecto a los gases inflamables, deben tenerse iguales precauciones que en la madera, no desprendiendose por la cara no expuesta gracias al poder aislante del tablero, excepto cuando éste está encerrado dentro de un elemento que se ensaye y del cual rome parte como aima.

El aislamiento térmico es excelente y gracias a él dichos tableros se utilizan como alma en la fabri. 


\section{CUADRO 7}

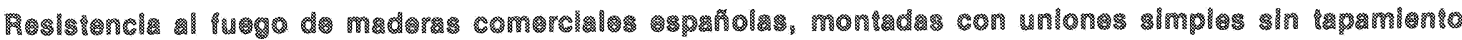

\begin{tabular}{|c|c|c|c|c|c|c|c|c|c|c|c|}
\hline \multirow[b]{2}{*}{ N. ${ }^{\circ}$} & \multirow[b]{2}{*}{ ESPECIE } & \multicolumn{2}{|c|}{$\begin{array}{l}\text { Cianllicación } \\
\text { (min.) RF }\end{array}$} & \multirow[b]{2}{*}{$\begin{array}{c}\text { Hume } \\
\text { dad }\end{array}$} & \multirow{2}{*}{$\begin{array}{c}\text { Frondosa } \\
0 \\
\text { conilura }\end{array}$} & \multirow[b]{2}{*}{ Empor } & \multirow{2}{*}{$\begin{array}{c}\text { Tabik } \\
0 \\
\text { Tablón }\end{array}$} & \multirow[b]{2}{*}{ Obsar. } & \multirow[b]{2}{*}{$\begin{array}{l}\text { T, amb } \\
\text { bient }\end{array}$} & \multirow[b]{2}{*}{$\begin{array}{l}\text { Cause del } \\
\text { fallo }\end{array}$} & \multirow{2}{*}{$\begin{array}{c}\text { Voll } \\
\text { perts } \\
\text { man / min }\end{array}$} \\
\hline & & $\begin{array}{r}\text { Contan: } \\
\text { huggo: }\end{array}$ & $\begin{array}{c}\text { Paxa } \\
\text { IIama }\end{array}$ & & & & & & & & \\
\hline 1 & $\begin{array}{l}\text { Pinus pinaster Alt. } \\
\text { (P. rodeno, P. negral) }\end{array}$ & 32 & 32 & 11,5 & Conlif. & 27 & Tabla & $\begin{array}{l}m_{m}=450 \\
T_{M}=65^{\circ}\end{array}$ & $20^{\circ}$ & Paso de llama & 0,75 \\
\hline 2 & $\begin{array}{l}\text { Pinus sylvestris L. } \\
\text { (PIno sllvestre) }\end{array}$ & 75 & 75 & 14,4 & Conif. & 67 & Tabion & & $20^{\circ}$ & Paso de llama & 0,85 \\
\hline 3 & $\begin{array}{l}\text { Fagus silvática L. } \\
\text { (Haya) }\end{array}$ & 47 & 47 & 11,0 & Frondos. & 40 & Tablon & & $22^{\circ}$ & Paso de llama & 0,78 \\
\hline 4 & $\begin{array}{l}\text { Fagus sylvática L. } \\
\text { (Haya). }\end{array}$ & 38 & 38 & 11,2 & Frondos. & 27 & Tabla & $\begin{array}{l}t_{m}=36 \\
T_{M}=50^{\circ}\end{array} \mid$ & $23^{\circ}$ & Paso de llama & 0,7 \\
\hline 5 & $\begin{array}{l}\text { Quercus robus L. } \\
\text { (Roble, carvallo) }\end{array}$ & 124 & 124 & 17,8 & Frondos. & 70 & Tablon & & $20^{\circ}$ & Paso de llama & 0,54 \\
\hline 6 & $\begin{array}{l}\text { Populus sp. } \\
\text { (chopo). }\end{array}$ & 65 & 65 & 12,8 & Frondos. & 70 & Tablon & & $20^{\circ}$ & Páso de llama & 1,03 \\
\hline
\end{tabular}

CUADRO

Reslstancla al fuego do tabloros do particulas In lonlluger

\begin{tabular}{|c|c|}
\hline Esposor (mm) & Sin Ignivgw AF (mim) \\
\hline $8-15$ & $7-20$ \\
\hline $15-22$ & $15-30$ \\
\hline $22-30$ & $25-40$ \\
\hline $30-50$ & $40-70$ \\
\hline $50-80$ & $60-115$ \\
\hline
\end{tabular}

FUENTE: INIA (OEPARTAMENTO DE MADERAS). cación de puertas con gran duración cortaluegos. En este aspecto la cota máxima exigida por temperatura en la cara no expuesta está asegurada y las variaciones dimensionales de la puerta son prácticamente despreciables.

Se pueden dar, en general, los siguientes valores de resistencla al luego para tableros sin ignifugar, obtenidos con un total de 20 tableros de tipo normal (cuadro 8).

\subsubsection{Ummacion del tablero}

Por sus propiedades mecánicas y de manipulación su empleo se extiende a múltiples usos de la edi. ficación, admitiendo recubrimientos de gran belle.

CUADRO

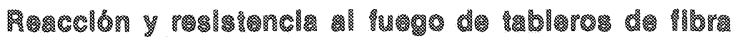

\begin{tabular}{|c|c|c|c|c|c|c|c|c|c|c|}
\hline \multirow{2}{*}{$\begin{array}{c}\text { maseor } \\
\text { mm. }\end{array}$} & \multirow{2}{*}{$\underset{\%}{\text { Humarad }}$} & \multicolumn{4}{|c|}{ Indicas } & \multicolumn{2}{|c|}{ CLASIFICACION } & \multirow{2}{*}{ 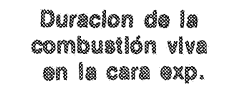 } & \multicolumn{2}{|c|}{ Valoc. de propagacion do la llama } \\
\hline & & 1 & 2 & h & 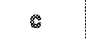 & Remeణ\&n & 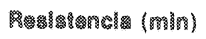 & & A Irख⿰纟: & En asmarte? \\
\hline 10 & 6,1 & 1,22 & 2,11 & 2,06 & 4,85 & $M-4$ & 14 & $4 \mathrm{~min} .15 \mathrm{seg}$. & 0,71 & $<0,2 \mathrm{~cm} / \mathrm{seg}$ \\
\hline 16,5 & 6,0 & 1,23 & 3,05 & 2,00 & 5,25 & $\operatorname{Mm}$ & 25 & $7 \mathrm{~min} .12 \mathrm{seg}$. & 0,66 & * \\
\hline 19,3 & 6,3 & 1,37 & 3,70 & 2,25 & 5,78 & $M-\infty$ & $30^{\prime} 20^{\prime \prime}$ & $7 \mathrm{~min} .57 \mathrm{seg}$ & 0,63 & s \\
\hline 25 & 6,2 & 1,48 & 4,64 & 2,00 & 4,05 & $M \propto 4$ & $3436^{\prime \prime}$ & $12 \mathrm{~min}, 30 \mathrm{seg}$. & 0,72 & 38 \\
\hline 30,3 & 6,8 & 1,12 & 5.46 & 2,00 & 7,28 & Mod & $4518^{s}$ & $14 \mathrm{~min}, 3 \mathrm{seg}$. & 0,67 & m \\
\hline 35 & 6,2 & 1,16 & 5,32 & 2,00 & 5,38 & $M \circ$ & 58 & $15 \mathrm{~min} .12 \mathrm{seg}$ & 0,80 & $\$$ \\
\hline 40,3 & 6,0 & 1,38 & 7,26 & 2,00 & 6,57 & M恶 & 62 & $22 \mathrm{~min} .15 \mathrm{seg}$ & 0,65 & $\$$ \\
\hline
\end{tabular}

FUENTE: INIA (DEPARTAMENTO DE MAOERAS). 
za, con ventaja sobre la madera maciza por no presentar exceslvo numero de luntas.

Su utilizacion en tabiques Ijos y móviles es exce lente, empleándose cllos solos o combinados con otros materiales en espesores desde 19 a $80 \mathrm{~mm}$. con resistencia al huego de cerca de $2 \mathrm{~h}$, pudiendo ser decorativo, alslante termico y fónico. Inclum so si el tablero está lonitugado (M-1 o M-2) per. mite embllecer la superficie.

Sobre su empleo en Fabricación de puertas ya he. mos hablado y en su puesta en obra deben sem guirse las mismas reglas generales que para la madera.

En cualquiera de estas utlizaciones la resistencia al fuego se ve disminuida notablemente sl existen juntas, por lo que es preciso cuidar éstas con detalle on los ensambles y ajustes. Los zócalos, viguetas y uniones deben tener, como es lóglco, una resistencia al tuego igual o superior al tam blero.

\subsection{EL TABLERO DE MIRAA}

El comportamiento al "uego y utlización de este tipo de tableros son muy similares a los de los tableros de particulas, pero su mavor finura en la composición del material base hace que su reac. ción al tuego sea más alta que en el de particum las, rayando con la Clase M-5, aunque sin llegar a ella.

Se hen ensayado tableros de varios espesores, sin lonifugar, arrolando los resultados sigulentes del Cuadro 9. Se observa una velocidad de paso de llama a través del tablero de $0,66 \mathrm{~mm} / \mathrm{min}$. que mantlene la constante de los elementos de made. ra, sl bien en este caso la tormación de capa de carbón superficial es más débil.

\subsection{PUERTAS DE MADERA Y DERUADOS}

En las puertas de madera, por ser esta muy ais lante, el grado para llamas y ol cortafuegos son práchicamente los mismos. Esto no ocurre con las metalicas, que si no están especiamente disenam das no pueden pasar de algunos minutos cortaluegos.

Una puerta plana de las que se utilizan nomalmente en el interior de nuestras viviendas, con materias sin tratar, ni holguras protegidas, consrulda con las dos caras de tablero de fibra o contrachapado y alma de sandwich de carton, no aguanta el incendio más que apenas 5 u $8 \mathrm{~mm}$. Los tableros con que están construidas, asi como los que consiluyen las mamparas de separación de ediliclos poseen una reacción al tuego destavom rable, pues el táblero contrachapado de las super ficies es extremadamente delgado y el alma de cartón es muy inilamable.

A estas puertas planas es suliciente con exigirles $1 / 4 \mathrm{~h}, 0$ a lo sumo $1 / 2 \mathrm{~h}$ de resistencla, dada la función que cumplen en el edificio.

Para conseguir el minimo exigido de $1 / 4 \mathrm{~h}$ es pre. ciso fabricar puertas de tablero de particulas normal con marcos o chasis de madera. Este tipo de tablero ya hemos visto que ralentiza la transm. slón de calor y con ellos pueda llegarse hasta resistenclas de $1 \mathrm{~h}$.

La obtención de una puerta de madera de $30 \mathrm{~min}$. cortafuegos no tlene ninguna dificultad si se culdan los herrajes y clerre témico de las cerradu ras. Para esta resistencia puede montarse ya un umbral o larguero que regule la holgura con el borde inferior del chasis de la puersa, ijazndolo en $203 \mathrm{~mm}$, si bien este elemento no es siempre aconsejable.

Resistencla cortafuegos de $1 / 2$ a $1 \mathrm{~h}$ precisan me jorar el chasls de la puerta y el marco y si se quiere llegar a $11 / 2 \mathrm{~h}$ se requieren diseños espe. clales o madera ignifugada o barnices ignifugos en la hoja con marcos y chasis culdados detalladamente y emplear, también, combinados con la madera, tableros de amiantomcemento o simllares. El espesor del chasis no debe ser interior a 60 milimetros sobre lodo el inferior, cercano al suelo.

Es preciso, como vemos, operar con los materia les base de madera ignilugados o tratados, con los cuales la resistencla al fuego de este tipo de puertas supera, fácimente, a la resistencla de puertas metellicas. Estas no pueden competir en ningún caso y en cuanto se refiere a resistencia al fuego, con las puertas de madera, a poco que en éstas se cuide el diseno. Por otra parte, el coste de tabricación de una puerta cortafuegos de ma. dera es aproximadamente la sexia parte de una metálica del mismo tlempo parallamas, y en esta unima ese tiempo contafuegos es muy difich conm seguirlo.

$$
\text { 순 숫 }
$$

